\title{
ADRENAL FEEDING IN CONDITIONS OF HYPERTHYROIDISM*
}

JOHN ROGERS, M.D.

Certain derivatives of the entire adrenal gland produce physiological reactions which differ materially from those of commercial adrenalin (Parke, Davis \& Co.).

The materials tested have been the nucleoproteins, the globulins and the coagulable albumens obtainable from aqueous extracts of this organ, and the non-coagulable "residue" of this extract. The latter is prepared as follows: After the precipitation by acetic acid of the nucleoprotein material from an aqueous extract of the hashed fresh (beef) adrenal glands, the filtrate is made slightly alkaline with $\mathrm{NaOH}$ and boiled to remove the alkali albumens; this filtrate is then again acidified and boiled to remove the acid albumens. There then remains a final dark-colored filtrate representing a non-coagulable somewhat hydrolyzed aqueous extract of the gland which, for lack of a better name, has been designated as the "adrenal residue." This is very rich in a pressor substance closely similar to epinephrin, but produces much more vigorous and lasting reactions, especially upon the stomach and pancreas, than do corresponding doses of adrenalin chlorid. An alcohol extract of the entire gland acts very similarly to this residue but not quite as intensively.

While studying these adrenal derivatives in comparison with adrenalin, it was found that they were all vasoconstrictors, apparently in proportion to their ephinephrin content, but differed decidedly from the latter material in that they all exhibited an inhibitory or "check" effect upon secretory activity, which was by no means in proportion to their epinephrin content. The results upon the pancreas, and particularly upon the stomach, were quite striking and seemed to indicate some clinical value in the adrenal nucleoprotein material which rather vigorously checks the total quantity of the gastric secretion and

* From the Johnston Livingston Fund for Experimental Therapeutics, Cornell University Medical College, New York City. 
also decreases its acidity and more or less inhibits gastric peristalsis. Corresponding doses of adrenalin showed few or none of these "check" effects, which seemed to be brought about by stimulation of the functions believed to be mediated by the terminal filaments of the sympathetic in contradistinction to those of the autonomic system. Incidentally, it was found that the hypodermatic administration of the adrenal residue or of an alcohol extract of the gland (made by evaporating the alcohol and adding water until the epinephrin content was equivalent to that of the residue) produced anorexia, vomiting and extreme prostration, and the frequent repetition of the dose was liable to cause death.

Because these derivatives of the gland showed such striking inhibition of the alimentary tract, it was decided to test their effects upon the iodin content of the thyroid, especially as our experiments seemed to indicate a marked degree of physiological antagonism between derivatives of the thyroid and those of the adrenal glands.

It has long been known that the thyroid gland in Graves' disease, or conditions of hyperthyroidism, contains less than the normal amount of about $0.5 \mathrm{mg}$. of iodin per gram of gland substance. Moreover, the severity of the disturbance seems more or less to correspond to the degree of the thyroid's iodin loss, or to the degree of its inability to hold iodin. Consequently, any medicament which experimentally makes the thyroid gain in iodin should be of some therapeutic value.

Dogs were employed in the tests, and it was first learned that the extirpation of one thyroid lobe resulted, in untreated, healthy animals, in a gain of some 20 per cent to 30 per cent of iodin in the remaining lobe. The maximum gain seemed to be reached at the end of three or four weeks. Intercurrent malnutrition, or disease like mange, apparently lessened this gain. At the time of the second operation no gross change in the size of the thyroid lobes could be detected. Only those animals were employed which seemed in vigorous condition, and in which both lobes on inspection, appeared to be alike. Under ether, one lobe was then removed, trimmed, weighed and its iodin content determined by the Kendall methods (1). In 
the preliminary tests the Riggs method (2) was used, but was later discarded in favor of the Kendall method which gives more uniform and trustworthy results. The same individual made all of these numerous iodin tests.

After excising one thyroid lobe from each of twelve selected animals, they were all then fed on a standardized diet consisting of cracker meal, evaporated milk, casein, lard, and bone ash, which was previously ascertained to maintain the nutrition and metabolism at a constant level. With this diet there was mixed daily for the first group of three, $0.016 \mathrm{gm}$. of adrenalin crystals (Parke, Davis \& Co.) ; for the second group, 2 c.c. of the adrenal residue (Schieffelin \& Co.), which contained an equal amount (0.016 gm.) of epinephrin-like material calculated by Folin's method (3) ; for the third group, $4 \mathrm{gm}$. of the adrenal nucleoprotein material (Schieffelin \& Co.). This latter was a rather arbitrary dosage, as the nucleoproteins contain only faint traces of epinephrin. The fourth group of three dogs received only the standard diet and were retained as "controls." At the end of forty-five days (or considerably later than the period at which the maximum gain in iodin had previously been ascertained to take place in the single remaining thyroid lobe) the second thyroid lobe was excised from all the animals, weighed and tested for iodin. There were noted some slight variations in a gain or loss of gross weight in these (second) excised lobes; but the changes could be traced to differences in the trimming of foreign tissue or to the variations in the amount of blood. These variations, however, seemed to bear no relation to the changes in the iodin content, which were quite marked.

The thyroids of the "control" animals gained the normal average amount of about 30 per cent in iodin. Those fed the pure (commercial) crystals of adrenalin showed a slight increase over this, but so little that it was judged to come within the limits of error. In short, the results of adrenalin feeding were negative. But the group which was fed the adrenal nucleoprotein material showed a considerable increase, or an average gain in iodin of about 50 per cent. Those fed the adrenal residue exhibited the greatest gain, or on an average of 70 per cent."

* A detailed statement of these experiments has been accepted for publication In the Am. J. Physiol. for March, 1922. 
Although this series is rather too small to permit any very sweeping conclusions, the results agree so perfectly with many rather rough preliminary tests that there can be little doubt of some direct influence of the adrenal gland upon the thyroid. This influence seems to be exerted not so much by the active principle of the medulla or epinephrin, as by a combination of this with some other material, possibly from the cortex or from the entire organ, for the adrenal nucleoprotein material, with its mere trace of epinephrin, seemed to make the thyroid gain much more iodin than did a great deal larger dose of the adrenalin crystals. The latter, as previously stated, show a practically negligible effect. That this influence of the adrenal nucleoproteins, and especially of the adrenal residue, upon the thyroid is one of "check," or inhibition, seems very probable because, as mentioned previously, of what is known of the iodin content of the thyroid in health and in conditions of its excessive secretory activity or hyperthyroidism. The administration of iodin to these patients is generally contraindicated beoause of the well known danger of producing a violent exacerbation of the disease. Apparently, the hyperthyroid gland is unable properly to metabolize the iodin which usually reaches it, and when this amount is increased the epithelium seems to proliferate and break down and form little or no colloid material.

As yet, we can only speculate as to how this takes place. But the formulation of a theory is legitimate and a very necessary precedent to an exact knowledge of the process which seems to involve the involuntary nervous system and its functions.

The chief symptoms of typical hyperthyroidism, or conditions in which there is supposed to be a superabundance of the thyroid product, are referable to hyperactivity of the autonomic group of these nerves. Practically the only evidence of sympathetic involvement is the tachycardia, which is supposed to depend upon irritation or stimulation by the thyroid, of the cardio-accelerator or a sympathetic nerve. But the vaso-dilatation, the sweating and the signs of gastrointestinal activity are all evidences, not of sympathetic, but of parasympathetic or vagus, stimulation. Furthermore, in the typical cases, the tachycardia is something more than a mere increase in the rapidity of the heart action. There is a certain degree 
of violence in the contractions which is very suggestive of vagus participation. Thus, clinically at least, the evidences of the disturbance in which the thyroid is believed to be abnormally active are all traceable to hyperactivity of the functions believed to be performed by the terminal filaments of the parasympathetic nerves.

Experimentally, the coagulable materials which can be isolated from the thyroid, when injected into animals, produce no appreciable immediate reaction. But the non-coagulable extracts, like one made with alcohol or a slightly hydrolyzed aqueous extract designated as the thyroid residue and prepared like the adrenal residue, are vigorous vasodilators and stimulants of the gastric and pancreatic secretions. These effects are produced apparently by direct stimulation of the terminal filaments of the parasympathetic or vagus group of nerves, which are believed in general to produce dilatation of blood vessels and increase in secretory activity (6). There are thus noncoagulable derivatives of the thyroid which excite immediate and definite physiological reactions.

Because these consist in stimulation of the functions believed to be performed by the parasympathetic or vagus portion of the involuntary nerves, and because this group shows marked activity in cases of typical hyperthyroidism, it is reasonable to believe that the thyroid product acts upon and through these particular nerve terminals and not through the sympathetic. Furthermore, because of the thyroid's well-known stimulating effect upon general metabolism, and because of the demonstrably stimulating effect of non-coagulable extracts of the thyroid upon some, if not all, parts of the parasympathetic system, the function of the product of the gland seems, broadly speaking, to be concerned with the production of energy in all the organs and tissues. In short, its function can be summarized as that of "drive."

Impulses which are antagonistic to those conveyed in the parasympathetic group of nerves seem to be discharged through the sympathetic terminals. Both are believed to be distributed to every organ, but to supply opposing impulses, or those which produce vasoconstriction and, in general, inhibition of functional activity. The action of the sympathetic nerves, with which the chromaffin tissue of the adrenal gland is so closely 
associated, is thus apparently opposed to the thyroid-autonomic or vagus system, and can be summarized as that of "check."

As stated previously, derivatives of the entire adrenal gland like the nucleoprotein material, and especially the adrenal residue, apparently intensify this check of the nerve impulse much more vigorously than the epinephrin of the medulla. All of these derivatives contain more or less epinephrin. The latter, therefore, seems to be in the living animal only a stable component of a possibly larger and more labile product. However this may be, the stimulation or intensification of the check nerve impulse by these derivatives of the (entire) adrenal gland, like the stimulation of the drive by the thyroid must be chemical. That is, the adrenal product after entering each cell must chemically restrain or "check" its metabolism, while the thyroid product on the other hand chemically accelerates or "drives" it. But each of these processes seems to depend upon the functional integrity of the nerve endings. For if these are lacking or are rendered functionless by poisons, as can be demonstrated in the stomach, the usual stimulation produced by the thyroid derivatives is absent. It is not as easy to destroy or paralyze the gastric sympathetic terminals and so demonstrate their importance for the action of the "check" derivatives of the adrenal. But these extracts have a stimulant effect upon the contraction of voluntary muscles, and this action is apparently exerted through the nerve end plate (5). The function of this organ can be completely abolished by fatigue and then the injection of the adrenal derivatives fails to excite a response. If, however, the fatigue is not complete, that is, if some response to electrical stimulation is still obtainable, the injection of the adrenal derivatives will immediately restore the vigor of the muscular contractions.

This fatigue experiment has considerable significance, not only in showing that the adrenal product probably acts through or upon the nerve terminals, but in suggesting the frequent origin of the hyperthyroid disturbance in a fatigue and consequent failure in functioning in the sympathetic or check mechanism. For if fatigue of the motor nerve end plate can abolish the influence of the adrenal product in voluntary muscles, fatigue of the involuntary or sympathetic nerve terminals in the viscera should interfere with or abolish their "check" influence. Be- 
cause of the presence of epinephrin in all of these adrenal derivatives and its well known affinity for the sympathetic terminals, there is every probability that the normal adrenal product contains at least an epinephrin-like substance, and consequently acts through or upon the sympathetic endings and so intensifies or stimulates their apparent check function. Fatigue of the involuntary nerves, though it has not actually been demonstrated, undoubtedly follows the same laws which govern the fatigue and abolition of function in the voluntary nerve endings. If then fatigue should occur in the sympathetic terminals around the thyroid epithelium, the adrenal product could not act and the normal check upon the metabolic activity of the thyroid would fail. The result should be hyperthyroidism.

Clinically, these disturbances are so much relieved by rest and so much intensified by fatigue, that fatigue of the sympathetic or check portion of the involuntary nervous system seems to be the primary and most frequent cause of these functional thyroid disturbances. They also seem to follow infections, and hence there is every probability of the occurrence of a toxic damage or destruction of the restraining sympathetic ter. minals. Their total loss of function would cause the adrenal product or adrenal feeding to be useless. If only partial, the adrenal product or adrenal feeding would be more or less effective, as suggested by the partial loss of function by fatigue in the experiments with voluntary muscle. If the loss of function is not complete, the injection of the adrenal derivatives, as stated previously, restores the full vigor of muscular contractions.

To recapitulate this theory for hyperthyroidism and the influence upon the iodin content of the thyroid by feeding the adrenal nucleoproteins or the adrenal residue: The thyroid epithelium is supposed to receive a parasympathetic (vagus) nerve supply which accelerates or "drives" its metabolism of iodin, and a sympathetic which "checks" it. The product of the thyroid containing iodin, after its utilization (or any ingested thyroid material or ingested iodin), returns to its epithelial source, where it enters the epithelium only through the intermediation of the parasympathetic nerve terminals. The ensuing chemical processes, or metabolism into a normal thyroid product, requires the normal functionation of the sympathetic or "check" nerve terminals in the thyroid through which the 
is high and especially when there are signs of excessive gastrointestinal activity, the nucleoprotein material with its low epinephrin content seems to be more valuable than the adrenal residue. The adrenal residue, with its high epinephrin content, on the other hand, seems valuable in the average case in which the blood pressure is low and the gastrointestinal activity not excessive. Although the thyroid product with its contained iodin is supposed to return to its source and not be destroyed or excreted, there ought theoretically to be some advantages in combining with the adrenal feeding the administration at the same time of small doses of iodin. Practically, it will often be noted that neither medicament alone will be as efficient as both together; but when taking iodin in any form, a patient with hyperthyroidism should always be warned that if the gland swells or "feels tight" the iodin should be stopped. If this gross change in the adrenal product alone can exert its restraint. If either the sympathetic terminals or the adrenal product fail, the thyroid's metabolism of iodin becomes defective or too rapid and hyperthyroidism is the result.

Of course, the most obvious criticism of this theory is that hyperthyroidism does not follow Addison's disease; but in this condition the sympathetic nerves are presumably intact and the adrenal product cannot be assumed to be the only material which affects the tropic functions of the sympathetic. Experimentally, the fact remains that feeding derivatives of the entire adrenal gland to dogs causes the animal's thyroid to gain noticeably in its iodin content. Why the feeding of one adrenal derivative should be more effective in causing the thyroid to gain in iodin than the feeding of another, is a matter for speculation. But, clinically, in cases of hyperthyroidism, when the blood pressure gland is neglected there is grave danger of an ensuing acute and often dangerous exacerbation of the hyperthyroidism. Very little iodin seems generally to be required. Three to five drops of the official tincture in a half glass of water once or twice a day is usually sufficient and may be too much.

The following case histories are illustrative.

CASE I. G. W., age 20, broker's clerk, after a prolonged period of hard work with late hours, was first observed in January, 1917, with typical exophthalmic goiter of three or four months' duration. There were: marked exophthalmos; considerable, pulsating, symmetrically 
enlarged goiter; pulse rate, 120 to 130 ; blood pressure, 120; weight, 145 lbs.

On Feb. 2, 1917, under local anaesthesia, both inferior thyroid vessels were tied, and on Feb. 9th, both superior vessels and a portion of the upper ends of both lobes were at the same time excised.

In April, the evidences of hyperthyroidism, with the exception of the goiter and some exophthalmos, had practically disappeared. The pulse rate was 80 , and the weight $165 \mathrm{lbs}$. In June, work was resumed, and later all the symptoms gradually returned.

Nov. 13, 1918. Under ether, two-thirds of each lobe was excised and all the vessels in sight were, of course, tied and cut. There was a somewhat stormy convalescence, and the patient'left the hospital at the end of a month improved, but still with marked signs of hyperthyroidism.

Jan. 9, 1919, there was moderate exophthalmos; the goiter, just after operation imperceptible, was now noticeable; the pulse rate was 100 to 110 ; blood pressure, 160; weight, 134 lbs. Weakness and diarrhoea were the chief complaints. Two 1-grain tablets containing 10 per cent of the adrenal nucleoproteins were then given every two hours. This form of adrenal feeding apparently had an immediately beneficial effect upon the diarrhoea, and the patient began to gain weight, which gradually increased during January and February to 150 pounds. The blood pressure fell from 165 to 145 , but the pulse rate continued from 110 to 120 .

In March, 1919, the adrenal residue in 10-drop doses every two hours was substituted for the adrenal nucleoproteins, and during the next month the pulse rate decreased to an average, while quiet, of about 90 . But there was still noticeable exophthalmos and a goiter which, in spite of the liberal excision in the second operation, was as large as at the beginning of the disease. Then iodin, in the form of 1-grain pills of iodid of iron, was given three times daily. Within a few days the general condition appeared practically normal, but the goiter was perceptibly larger and firmer. The iodid of iron was, therefore, ordered to be discontinued. Two weeks later this patient was found with a rather violent exacerbation of his hyperthyroidism, apparently due to the iodin, which had been continued in spite of directions to the contrary. There was a temperature of 102; the pulse rate was 150 to 160 ; the blood pressure, 160, and the sweating and diarrhoea had returned.

During the next two weeks with feeding the adrenal nucleoprotein tablets, which seem particularly beneficial for the cases with diarrhoea, the symptoms gradually improved. When the gastrointestinal disturbance had subsided, feeding with the adrenal residue was resumed in 10 or 12 drop doses every two hours.

On May 15th, the pulse rate was about 90 , but irritable; the blood pressure, 140; and the exophthalmos and goiter, noticeable. The 1-grain iodid of iron pills were then given one daily, but at the end 
of ten days, although the hyperthyroid signs had nearly disappeared, the goiter began to swell and was accompanied by a feeling of pressure in the neck. The iodin was, therefore, immediately stopped until the uncomfortable cervical sensation had disappeared, and the goiter felt soft. By a little cautious experimenting, it was finally found, after another two weeks of the feeding with the adrenal residue, that the 1-grain iodid of iron pill could be taken every second day with apparent great advantage and without any subsequent swelling and "tightening" of the goiter.

If the adrenal feeding were omitted for two or three days the goiter immediately began to enlarge and feel uncomfortable. Apparently, the iodid had to be accompanied with the adrenal feeding to insure progress, and during June it was found that the iodid could be taken daily. In July and August the combination of iodin and adrenal feeding gradually seemed to complete the cure of all the symptoms except the exophthalmos and the goiter. The latter, however, had entirely disappeared in October, and the following month there was no exophthalmos and the medication was omitted. In January, 1920, the weight had reached 170 , and no abnormality other than the cervical scars could be detected. This patient has continued well.

CASE II. Mrs. A. M., age 42 , presented a typical case of rather severe exophthalmic goiter, with slight exophthalmos, and only a just perceptible and symmetrical, firm enlargement of the thyroid. There was some pigmentation of the skin, considerable emaciation, much nervous irritability and frequent bowel movements. The weight was $86 \mathrm{lbs}$., pulse rate was 110 to 120 , and the systolic blood pressure was 215. This patient had been confined to bed with a general sedative treatment for several weeks. Jan. 18, 1916, two 1-grain tablets containing 10 per cent of the adrenal nucleoproteins were given every two hours with an almost immediate relief of the frequent bowel movements and a more gradual decrease in the tachycardia.

- On Feb. 15, the pulse rate averaged about 90 , the weight had increased to 92 pounds, and the systolic blood pressure had fallen to 150. The goiter was softer, but the exophthalmos, unchanged. Operation was declined, as the improvement seemed to promise a recovery.

In September, 1916, after a period of considerable comfort and apparently following a few weeks of strenuous work, there was an exacerbation of the hyperthyroidism which was accompanied by a systolic blood pressure of 210. Feeding for about three weeks with the adrenal nucleoprotein material again seemed to reduce this to between 140 and 150 , and also all the other symptoms. At the same time, the weight increased. One-grain iodid of iron pills were then added, one daily, to the adrenal feeding, and at the end of the month practically all of the symptoms had disappeared. This patient, later, again relapsed and again improved, and operation under the circumstances seems to offer little hope of a permanent cure. 
CASE III. F. N., age 38, a salesman, presented typical exophthalmic goiter of several weeks duration. March 10,1921, moderate exophthalmos; considerable symmetrical goiter, circumference of neck $15 \frac{1}{2}$ inches; weight, 150 lbs.; pulse rate, 120; blood pressure (systolic), 160 , were noted. He was given the 1-grain pills of iodid of iron, three daily, and warned to stop them if they caused swelling of the neck or any cervical discomfort. At the end of two days both the swelling and discomfort were noted, and, after discontinuation of the iodin, these signs soon disappeared.

March 22, 1921, the adrenal residue in 10-drop doses every two hours was then administered, with rapid improvement, especially in the subjective nervous irritability. The pulse rate was 90 , blood pressure (systolic), 150; weight, 152 pounds. There was a slight decrease in the exophthalmos and goiter.

April 15, 1921, the patient returned after a month's rest in Atlan'tic City. The pulse rate ranged from 80 to 90 ; blood pressure was 145; weight, 155 pounds. One-grain iodid of iron pills once daily were now added to the adrenal residue, with warning as to the repetition of its possible ill effects.

May 23, 1921, gain much more than before taking the iodin was noted. The pulse rate was 82 ; blood pressure, 132; weight, 163 pounds. The circumference of the neck was $14 \%$ inches, showing marked decrease in goiter; the exophthalmos was less pronounced. The patient returned to work.

Sept. 12, 1921, the medication of the preceding month was discontinued and again marked and typical symptoms developed, but there was no loss of weight. Operation was advised, but at present the patient is again trying rest and the combination of iodid of iron with the adrenal residue.

\section{SUMMARY}

Experimentally, the feeding by mouth to dogs of derivatives of the entire adrenal gland, especially the adrenal nucleoproteins and a slightly hydrolyzed aqueous extract known as the adrenal residue, causes the animal's thyroid to gain from 50 to 75 per cent or more in its iodin content within a few weeks.

Feeding with corresponding amounts of adrenalin crystals is without appreciable effect upon the thyroid.

In conditions of hyperthyroidism the thyroid gland contains less than the normal amount of iodin per gram of gland substance.

The failure thus indicated of the thyroid to retain its normal amount of iodin apparently is due to a defect in the metabolism of iodin by the thyroid epithelium, and this defect is the probable biochemical cause of the disturbance. 


\section{ADRENAL FEEDING IN HYPERTHYROIDISM}

The defect in the thyroid epithelial metabolism is theoretically dependent upon some preceding defect in the chromaffin, or automatic "check" system, and primarily begins in a failure in functioning of the thyroid terminals of the sympathetic nerves. It seems probable that the adrenal product acts through or upon these nerve terminals and, therefore, the functional integrity of these terminals is essential for the success of adrenal feeding.

Feeding with adrenalin, or the so-called active principle of the medullary portion of the adrenal gland, has no appreciable effect upon the iodin content of the dog's thyroid, and in human hyperthyroidism does not relieve the symptoms and may often intensify them (Goetsch test).

Three illustrative cases are cited.

\section{BIBLIOGRAPHY}

1. Kendall, E. C.: J. Biol. Chem. (Balt.), 1914, 19, 25.

2. Riggs: J. Am. Chem. Soc., (Easton, Pa.), 1909, 31, 710.

3. Folin, O.: J. Biol. Chem. (Balt.), 1913, 13, 477.

4. Rogers, J., Rahe, Jessie M., Fawcett, G. G. \& Hackett, G. S.: Am. J. Physiol. (Balt.), 1916, 39, 345; Rogers, J., Rahe, Jessie M. \& Ablahadian, E.: Ibid., 1919, 48, 79.

5. Rogers, J., Coombs, Helen C. \& Rahe, Jessie M.: Am. J. Physiol. (Balt.), 1918, 45, 97 . 